\title{
Study on Electric Power Enterprise Operation Risk Measurement Based on Blind Number Theory
}

\author{
Fusen Zhong ${ }^{1 \text { a }}$, Shenyou Zhang ${ }^{2}$ \\ 1.State Grid Energy Reseach Institute,Beijing,China \\ 2. Research Institute for Fiscal Science, Hefei,Anhui. \\ aemail:zhongfusen@sgeri.sgcc.com.cn
}

\begin{abstract}
Keywords: Power Enterprise; Factor Analysis Method; Blind Number Analysis; Operational Risk. Abstract. This study adopts questionnaire survey to analyze and comb operational risk of the electric power enterprise, and construct the index system of operational risk of electric power enterprise. Then adopt the factor analysis method and blind number theory to screen and quantify the key indicators of operational risk.
\end{abstract}

\section{Introduction}

With the development of economic technology, the social basis role of the power is increasingly prominent, while safe operation of the power companies is affected by national policy adjustment, the socio-economic development, the international economic situation changes, technological advances, demographic changes, environmental changes and other factors. And these influences have many characteristics, such as uncertainty, contingency, randomness and difficult quantification, which bring operations management of power enterprises a huge challenge. In addition, due to the limitations of enterprise resources, enterprises cannot conduct a comprehensive monitoring and response measures to all risk factors that they face. Therefore, based on comprehensive combing business operations risk, enterprises identify the key impact indicators and further study and quantify them to provide support for enterprise risk management decision-making and risk plans establishment. Sequentially, it helps to improve the efficiency and effectiveness of enterprise management and has a positive effect on implement and realization of enterprise strategies.

\section{Literature Review}

As research moves along, risk management concepts are continuously enriched. Risk management study is divided into three stages: the traditional risk management, modern risk management and comprehensive risk management. In the traditional risk management stage, the study objects primarily are credit risks and financial risks. Risk Management of this stage limits to certain variable and local variable, so it fails to systemically and globally consider more complex risk problems; in the modern risk management stage, risk management focuses on the combination of multiple variables and scholars proposed an idea about "risk portfolio, the risk of cross-sectoral integration and enterprise-wide risk management". In 1952, Markowitz published "Portfolio Selection" theory, which assumes the investment risk is an uncertain part of investment returns. Such uncertainty can make use of the variance and standard deviation of statistical theory to measure and it opens up new path for the study of the financial risk. At the same year, Sharp proposed asset pricing theory (CAPM) on the basis of the portfolio theory and Ross proposed asset pricing model on the basis of portfolio theory and pricing theory, forming arbitrage pricing theory. In 1973, option pricing formula greatly enhanced the level and strategy of risk management; since 1998, Theory of Enterprise Risk Management(ERM) and the Theory of Global risk management(GRM) have been developed and applied widely. Comprehensive risk management theory believes that enterprises should comprehensively arrange the risk they face. First, establish a set of metrics to take a coherent, accurate, and timely measure to enterprise risk. Secondly, establish specialized risk control department within the finance organization working to prevent and resolve risks. 
Risk Management stars late in our country and it mainly introduced foreign risk management ideas and management methods in the early. Lei Yang and Yizhen ZHANG (1998) once applied factor analysis to diagnosis and identification process of logistics and financial risk of agricultural products. Since a large number of risk factors cannot be quantified and there is a high correlation and complexity between the risk factors, it is difficult to analyze the complex relationship between risk factors just from a qualitative point of view. By making the use of factor analysis, a number of risk variables are condensed into the core factor variables, reduce the complexity of quantitative assessment of risk and to some extent weaken multiple correlations between the indicators, to make results of quantitative assessment close to the actual. Liang Cai, Tieyuan Xiang, Hui Huang (2003) and other researchers believed that blind number theory can not only be used to describe the uncertainty, but also can be applied to deal with application and planning risk, particularly issues with supply and demand concept. They also select the electric energy supply and demand system, using blind number theory, to provide reliability evaluation model and relevant indicators of the power system. Yong Chang, Hongchun Shu, Wentao Zhu (2007) and other researchers used blind number modeling method to predict power grid planning load. According to the randomness, fuzziness, uncertainty and etc. of risk information, with the help of the blind information theory, risk information will be quantified. Lingjiao Yin, Qingqin Xue, Guohua Li (2011) and other researchers studied risk assessment of a power supply bureau, applying blind number theory, expert evaluation scoring can quantify the uncertain information, such as the severity, the possibility of occurrence, controllability and etc. Finally, calculate risk progressive number by making use of the formula.

In summary, based on the combing of operational risk of power enterprises, we use factor analysis method and blind number theory to extract the core risk indicators of operational risk management and to further quantify the key risk indicators, which provide support to risk management decisions and risk preparedness plans for enterprises.

\section{Operational risk quantification theory and model design}

Risk quantification is the difficulty of risk management. With the deepening of risk management research, academia and industry recognize the limitations of the individual risk factors management. There are potential linkage relationships between these potential risk factors, thus the implementation of comprehensive risk management will be able to improve risk management performance level. In order to improve the efficiency and effectiveness of risk management and maximum the effect of limited resources, therefore, we should use methods to select major risk factors and quantitatively assess the main risks on the basis of comprehensively combing of risk factors, which provide support to risk management decisions and risk preparedness plans for enterprises.

\section{Risk quantification theory}

\section{Factor Analysis}

Factor analysis method refers to a multivariate statistical analysis method, which studies dependencies of internal index correlation matrix and refines some variables with overlapping information and complex relationship into a handful of unrelated comprehensive factors. The basic idea is: we group variables according to their correlations to ensure high correlations between variables within the same group, but different groups of variables are not relevant or less relevant. Each group of variables represents a basic structure, says common factor.

Assume that $\mathrm{n}$ assessment objects constitute a sample set $\left\{N_{1}, N_{2} \mathrm{~L} N_{n}\right\}$. And suppose that the evaluation system has a total of $m$ assessment indexes that are defined as variable $\left\{X_{1}, X_{2}\left\llcorner X_{n}\right\}\right.$. After non-dimensional raw data, the raw data matrix is obtained:

$$
X_{m \times n}=\left[\begin{array}{l}
x_{11} \cdots x_{1 n} \\
x_{m 1} \cdots x_{m n}
\end{array}\right]
$$


The correlation matrix will be $R=X X^{T}$. Thus, solve the characteristic equation $|R-\lambda I=0|$ of the correlation matrix to obtain eigenvalues $\lambda_{1} f \lambda_{2} f \lambda_{3} f \lambda_{m} \geq 0$ and eigenvector matrix $A$, where $A$ is an orthogonal matrix.

Suppose that the main factor matrix is $F=A^{\prime} X$. In order to simplify evaluation system in factor analysis, it is usual to only choose $\mathrm{P}$ main factors (where $\mathrm{p}<\mathrm{m}$ ) to represent the overall information. Therefore, generally decompose $\mathrm{A}$ and $\mathrm{F}$ into two portions in factor analysis:

$$
\begin{aligned}
& A_{m \times m}=\left[A_{m \times p}^{(1)} A_{m \times(m-p)}^{(2)}\right] \\
& F_{m \times m}=\left[F_{m \times p}^{(1)} F_{m \times(m-p)}^{(2)}\right]
\end{aligned}
$$

The factor model is:

$$
X-A F-A^{(1)} F^{(1)}+A^{(2)} F^{(2)}
$$

where $A^{(1)} F^{(1)}$ is the part that $\mathrm{p}$ main factors can explain. And is the residue that contains a few information, so it can be omitted. Therefore, the model can be specifically expand to:

$$
\begin{aligned}
& X_{1}=a_{11} f_{1}+a_{12} f_{2}+\mathrm{L} a_{1 p} f_{p} \\
& X_{2}=a_{21} f_{1}+a_{22} f_{2}+\mathrm{L} a_{2 p} f_{p} \\
& X_{3}=a_{31} f_{1}+a_{32} f_{2}+\mathrm{L} a_{3 p} f_{p}
\end{aligned}
$$

where $f_{1}, f_{2} \mathrm{~L} f_{p}$ are the main factors. They are unobserved latent variables that respectively reflect a certain aspect of information: ${ }^{a_{i_{j}}}$ is factor loading coefficient which is the load of $i$ index on the $\mathrm{j}$ factor. If an index plays a very important role in a factor, the factor loading coefficient is large, and vice versa.

Finally, score evaluation model of the sample is:

$$
S=\sum_{i=1}^{p} d_{1} f_{1}=\sum_{i=1}^{p} \sum_{j=1}^{m} d_{i} b_{j i} x_{j}
$$

where $d_{i}$ is factor contribution rate, $f_{i}$ is factor score, and $b_{j i}$ is factor score coefficient.

After determining the factor model, we need to measure the risk of a single factor. Factor analysis addresses order of importance of the risk factors, and screens multiple risk factors in terms of importance to provide a basis for risk quantification and assessment.

\section{blind number theory}

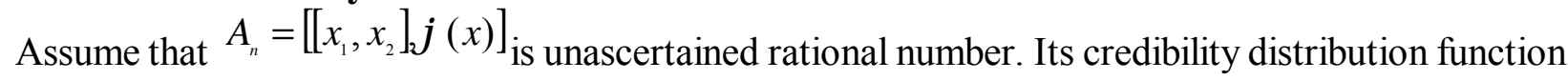
$\phi(x)$ is a real function and $i=1,2 \mathrm{~L} n, 0 \leq a_{i} \leq 1, a=a_{1}+a_{2}+\mathrm{L} a_{n} \leq 1$, where shows that $\varphi(x)$ takes non-zero values at a finite points and takes zero value at other points. Since the probability of occurrence of each risk is uncertain, their actual observed values (an expert experience value in this paper) usually do not fall on one determined point xi , and it is more reasonable to be near xi and intervals with confidence $\alpha \mathrm{i}$. Thus, expand the domain of credibility distribution function from the real domain to the interval gray number set. Blind number is defined as a certain interval type $G(\mathrm{I})$ value of gray function on $[0,1]$. According to the definition of the blind number, it substantially is a credibility function of interval distribution.

Suppose G(I) is a set of interval gray numbers that is composed of a series of gray interval xi , so xi $\in \mathrm{G}(\mathrm{I})$. If the general real numbers $\alpha \mathrm{i} \in[0,1], \mathrm{i}=1,2, \ldots, \mathrm{n}, \mathrm{f}(\mathrm{x})$ is a gray function defined on $\mathrm{G}(\mathrm{I})$ and:

$$
f(x)= \begin{cases}\alpha_{i}=x_{i} & (i=1,2, \ldots n) \\ 0 & \text { other }\end{cases}
$$

where $x i(i=1,2, \ldots, n)$ are $n$ gray intervals that are mutually unequal and $\alpha 1+\alpha 2+\ldots+\alpha n=\alpha \leq 1$. We name that $f(x)$ is a blind number, $\alpha \mathrm{i}$ is the credibility of $\mathrm{xi}$ of $\mathrm{f}(\mathrm{x})$, ais the total credibility of $\mathrm{f}(\mathrm{x})$ and $\mathrm{n}$ is 
the order of $\mathrm{f}(\mathrm{x})$. According to the definition of the blind number, we can describe the probability of the occurrence of risk as possible values and credibility of intervals of the probability of occurrence.

\section{Operational risk quantification model design}

According to the SASAC for comprehensive risk management requirements, risk management is divided into five categories: strategic risk, financial risk, market risk, operational risk (core business and management), and legal risk. The operational risk management requires relating to research and development, marketing, organizational structure and culture, derivatives business process, security and environmental protection and etc. As a pillar of national economy and the people's livelihood, electric power enterprises' environment is complex, involved multifaceted operational risk factors, therefore need to analyze internal and external environment of electric power enterprises and structure risk indicators.

Combined with these ideas, first, do factor analysis on risk indicators to identify the main factor, and then conduct blind number assessment of the main risk indicators. This study proposes U-shaped model of operational risk management of electric power enterprises, as shown in Figure 1.

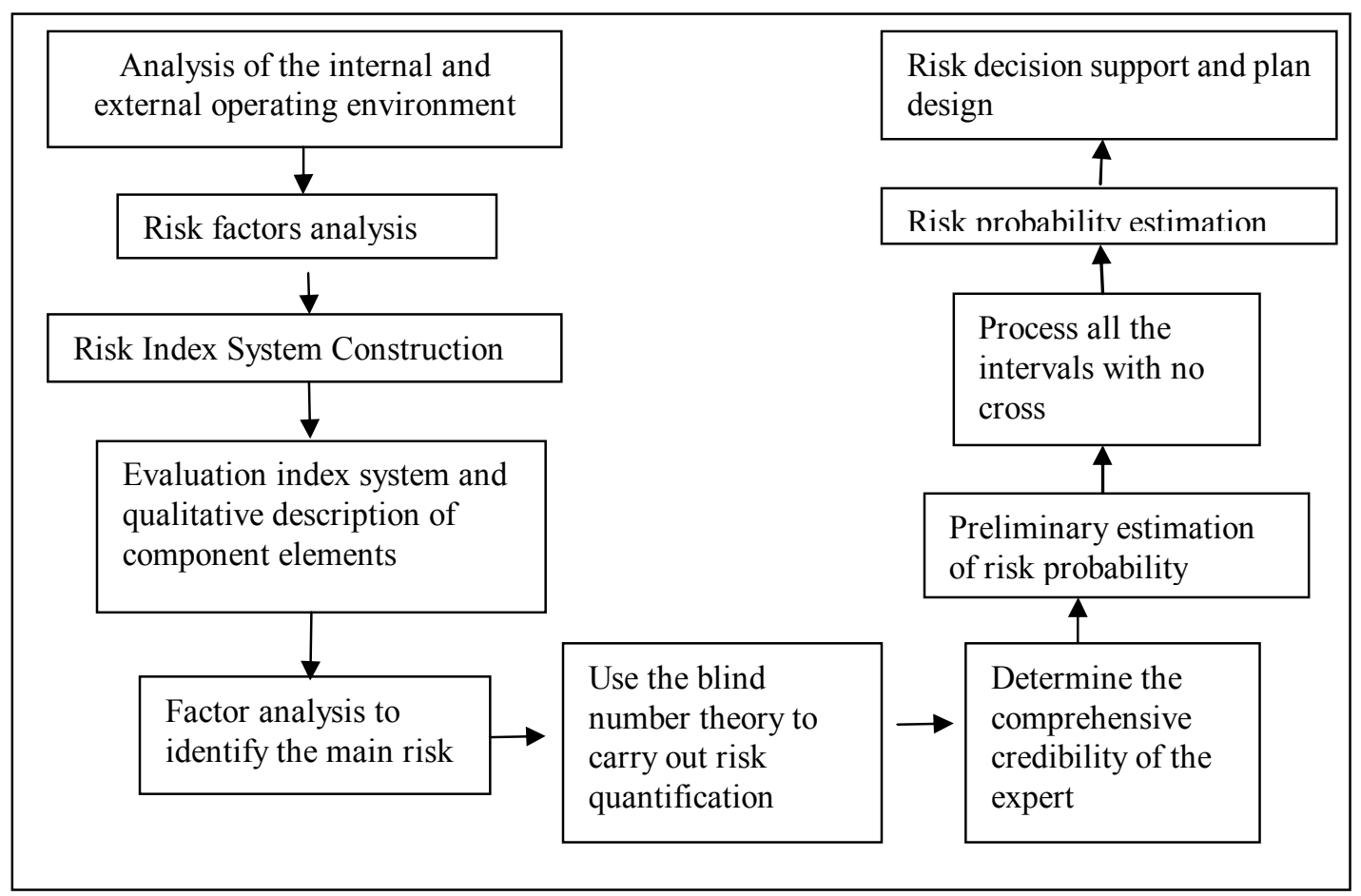

Figure 1 U-process Model of Electric Power Enterprise Operational Risk Quantitative Research

\section{Empirical study on electric power enterprises operational risk quantification}

\section{Operational risk questionnaire design}

The questionnaire includes risk type, description, severity, frequency of occurrence, status, main factors of risk and so on. the questionnaire will divide the risk that the company confront into five categories, namely strategic risk, financial risk, legal risk, operational risk and market risk, the severity of the risk has five cases: slight, small, general, larger, severe, and are respectively quantized value of 1 , 2, 3, 4 and 5; the risk of frequency considers three cases: low, medium, high, and are respectively quantified to the value of 1,2 and 3; the risk status taking three cases into account: had occurred, is occurring, will take place.

We also conduct an investigation on demand of risk management system. we propose measures to strengthen the company's risk management in nine aspects: establish a risk management organization system in the company syste, establish a unified risk management information system, regularly report important business activities, clear the link of risk management in the business process, carry out propaganda of concept of risk and establishment of risk culture, establish risk management positions at 
departmental level, involve risk management into performance evaluation content, submit risk analysis report while making major decisions, carry out risk management training and develop a team. We sincerely invite each institution to assess in the aspects of two dimensions: the urgency and importance, where the urgency takes three cases into account: the general, urgent and very urgent, and the importance takes three cases into account: the general, important and very important.

\section{Quantify key risk factors}

\section{Screening of key risk factors}

After analyzing the questionnaires, we summarize and select Governance structure risk, Management capability risk, Grid investments risk, Financial risk, Industry risk, International operations risk, Mergers and Acquisitions risks, Technological innovation risk, Management Innovation risk, Brand management risk, Public relations risk and Corporate culture risk and etc.

First, invite relevant experts to score the importance of risk object. Give the most important risk 1 point, descend importance of the risk and increase the score meanwhile, with 7 points to the least. Do Bartlett ball type test and KMO test on 12 variables to test whether data is suitable for factor analysis, and the test results show that there is a strong correlation between the original variables.

Second, verify dependence of variable on common factors. Calculation results communalities of variables show that common factors can well contain variables; communalities of selected variables are more than $80 \%$ and the extracted factors contain most of information of the original variables. Further public factors characteristic root and variance analysis on common factors shows that a combination of common factors includes $89.65 \%$ of the variances that can explain the original variables.

Finally, according to the characteristic root values, select and rotate factor loading matrix, which is resulted in the following table:

Table 1 rotated factor loading matrix

\begin{tabular}{|l|l|l|l|l|}
\hline \multirow{2}{*}{\multicolumn{1}{c|}{ Variable }} & \multicolumn{4}{c|}{ Factor } \\
\cline { 2 - 5 } & \multicolumn{1}{|c|}{ F1 } & \multicolumn{1}{c|}{ F2 } & \multicolumn{1}{c|}{ F3 } \\
\hline Governance structure risk & -0.9134 & -0.147 & 0.4523 & 0.0556 \\
\hline Grid investments risk & -0.0434 & -0.5332 & -0.2356 & -0.4356 \\
\hline Management capability risk & 0.6435 & 0.3057 & 0.4557 & -0.5467 \\
\hline Brand management risk & -0.6596 & 0.0200 & -0.448 & 0.5673 \\
\hline Industry risk & -0.4536 & 0.7778 & -0.2456 & 0.7654 \\
\hline International operations risk & 0.7563 & -0.0840 & -0.0892 & 0.9454 \\
\hline Mergers and Acquisitions risks & 0.2345 & -0.3932 & 0.0743 & 0.9435 \\
\hline Corporate culture risk & 0.5442 & 0.0435 & 0.4536 & 0.6563 \\
\hline Management Innovation risk & 0.4543 & 0.3412 & 0.5463 & 0.4535 \\
\hline Financial risk & 0.3256 & 0.7345 & 0.7637 & 0.5436 \\
\hline Public relations risk & -0.4256 & 0.5462 & 0.5473 & 0.7562 \\
\hline Technological innovation risk & 0.5462 & 0.5463 & 0.7847 & 0.3535 \\
\hline
\end{tabular}

The results obtained from the data in the table are as follow:

Factor F1 and variable X1, X3 have a strong correlation, indicating that it generally has a high coupling with governance structure and capability risk in strategic risk; factor F2 and variable X2, X5, $\mathrm{X} 6, \mathrm{X} 7, \mathrm{X} 10$ have a strong correlation, indicating that it high couples with power grid investment risk, industry risk, the risk of international operations, mergers and acquisitions risk, financial business risk in the strategic risk; factor F3 and variable X9, X12 strong positive correlation, indicating that it has a high coupling with technological innovation, management innovation risk in strategic risks; factor F4 and X4, X8, X11 have a strong positive correlation, indicating that it high couples with brand management risk, the risk of public relations, corporate culture risk in strategic risk.

To evaluate the impact of each risk factor on the power grid operation, it is necessary to calculate the composite score for each risk variable. The calculation method is: use the proportion of the contribution rate to the variance of each factor accounted for a total contribution rate of four comprehensive factor variance to weight by the sum. 
The formula is: $\mathrm{F}=(9.00 \% * \mathrm{~F} 1+28.20 \% * \mathrm{~F} 2+34.45 \% * \mathrm{~F} 3+18.0 \% * \mathrm{~F} 4) / 89.651 \%$, and the results are in the table below:

Table 2 scores of factors:

\begin{tabular}{|l|r|r|r|r|r|}
\hline \multirow{2}{*}{ Variable } & \multicolumn{4}{|c|}{ Factor } & \multicolumn{1}{l|}{$\begin{array}{l}\text { Composite } \\
\end{array}$} \\
\cline { 2 - 7 } & F1 & F2 & F3 & F4 & \multicolumn{1}{l|}{ Score } \\
\hline Governance structure risk & -0.435 & 0.145 & 0.343 & 0.435 & 0.488 \\
\hline Grid investments risk & 0.435 & 0.345 & -0.144 & 0.435 & 1.071 \\
\hline Management capability risk & 0.234 & -0.342 & 0.342 & 0.123 & 0.357 \\
\hline Brand management risk & 0.412 & 0.124 & 0.562 & 0.622 & 1.72 \\
\hline Industry risk & -0.425 & 0.345 & 0.341 & 0.341 & 0.602 \\
\hline International operations risk & 0.145 & 0.432 & -0.341 & 0.542 & 0.778 \\
\hline Mergers and Acquisitions risks & 0.345 & 0.631 & 0.521 & 0.134 & 1.631 \\
\hline Corporate culture risk & 0.452 & 0.653 & 0.345 & 0.342 & 1.792 \\
\hline Management Innovation risk & 0.245 & 0.342 & 0.632 & 0.532 & 1.751 \\
\hline Financial risk & 0.234 & 0.324 & 0.532 & 0.442 & 1.532 \\
\hline Public relations risk & -0.634 & 0.424 & 0.234 & 0.234 & 0.258 \\
\hline Technological innovation risk & 0.435 & 0.323 & 0.342 & 0.342 & 1.442 \\
\hline
\end{tabular}

Based on the above description and analysis, twelve importance of operational risks in strategic risk of power grid can be more accurately re-positioned as: cultural risk, management innovation risk, brand management risk, mergers and acquisitions risk.

Quantify key risk factors

After determining the key risk factors, we need to quantify key risks. Brand management risk, for example, we make the use of blind numbers to quantify risk.

(1) Invite 10 experts to form a risk assessment expert group to assess risk factors that affect the company risk indicators:According to the prestige, knowledge level and work experience of 10 experts, make the use of fuzzy evaluation method to calculate the reliability of each expert. Analog computation result of credibility vectors of each expert in this group is $(0.9,0.9,0.85,0.9,0.85,0.8,0.8,0.85,0.75,0.7)$. Then based on blind number theory calculation formula, determine each expert on the comprehensive credibility of strategic risk expert group is: $\alpha 1=0.108$, $\alpha 2=0.108, \alpha 3=0.102, \alpha 4=0.108, \alpha 5=0.102, \alpha 6=0.096, \alpha 7=0.096, \alpha 8=0.102, \alpha 9=0.09$ , $\alpha 10=0.084$. Thus the comprehensive credibility of strategic risk expert group is $\alpha=0.83$.

(2)Expert group of risk assessment of managing innovation estimates the probability of occurrence of managing innovation risk in the process of power grid operation based on working experience in the management of innovation process grid operators to risk

Table 3 estimation of occurrence of power grid brand management risk:

\begin{tabular}{|l|l|l|l|}
\hline expert & $\begin{array}{l}\text { Probability of } \\
\text { Brand Management } \\
\text { Risk }\end{array}$ & expert & $\begin{array}{l}\text { Probability of } \\
\text { Brand } \\
\text { Management Risk }\end{array}$ \\
\hline expert a & {$[0.05,0.10]$} & expert & {$[0.10,0.20]$} \\
\hline expert b & {$[0.10,0.15]$} & expert & {$[0.10,0.20]$} \\
\hline expert c & {$[0.05,0.10]$} & expert & {$[0.10,0.15]$} \\
\hline expert d & {$[0.05,0.10]$} & expert & {$[0.10,0.15]$} \\
\hline expert e & {$[0.10,0.15]$} & expert & {$[0.10,0.20]$} \\
\hline
\end{tabular}

There is a range of overlapping phenomenon occurs as shown in Table 2, between the estimation result of each expert about the occurrence of brand management risk. Based on blind number theory, reorder endpoints of occurrence of the brand management risk in order of size, so that no cross-section between the new sequence. The results of reordering brand management risk are $0.05,0.10,0.15$, and 0.20 . The new intervals are $[0.05,0.10],[0.10,0.15],[0.15,0.20]$. 
According to the formula 5, the confidence values of new divided intervals are $\beta 1=0.318, \beta 2=0.540$ , $\beta 3=0.142$, resulting in brand management risk distribution:

Table 4 probability distribution of occurrence of brand management risk:

\begin{tabular}{|l|l|}
\hline confidence & $\begin{array}{l}\text { probability of occurrence of } \\
\text { brand management risk }\end{array}$ \\
\hline 0.318 & {$[0.05,0.10]$} \\
\hline 0.542 & {$[0.10,0.15]$} \\
\hline 0.142 & {$[0.15,0.20]$} \\
\hline
\end{tabular}

Finally, calculate the expected value of occurrence of brand management risk, as well as the probability of risk ${ }^{x=\frac{1}{a}\left(\Theta \sum_{i=1}^{\infty} a_{i} x_{i}\right)}$, in this case $\mathrm{x}=0.116$. That is, the probability of risk occurrence may be $11.6 \%$.

\section{Construct criteria for the risk classification}

The loss that brand management risk leads to is very high, according to the company's operational experience to build a quantitative standard of the risk of management innovation, as shown in the table below:

Table 5 Brand management risk quantification standards table

\begin{tabular}{|l|l|}
\hline $\begin{array}{l}\text { Brand Management Risk } \\
\text { Quantification }\end{array}$ & status \\
\hline$[15,20]$ & High risk \\
\hline$[10,15]$ & Higher risk \\
\hline$[5,10]$ & Medium risk \\
\hline$[2,5]$ & Lower risk \\
\hline$[0,2]$ & Low risk \\
\hline
\end{tabular}

In this case, the probability of brand management occurrence is $10<11.46<15$, which belongs to a higher risk variable. Therefore, in the course of the company operations, we should actively adopt corresponding measures and take professional measures to address the problem that is triggered by brand management.

\section{The key risk distribution map}

According to blind number analysis, we continue to quantify the other major risk factors, such as corporate culture risk, brand management risk and the risk of merger and reorganization, and calculate the probability of their occurrence, in order to draw out the risk occurrence probability diagram, as shown below:

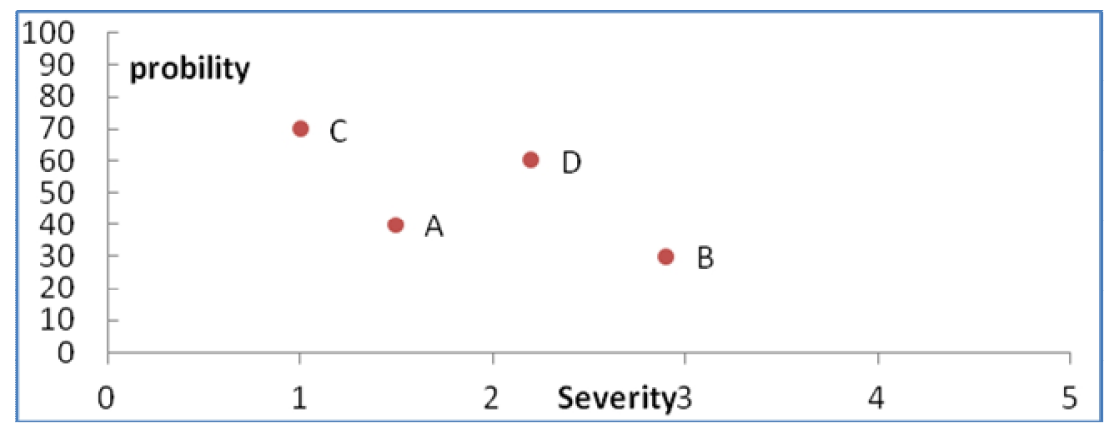

Figure 2 Key Risk Map Distribution Map

In figure2, we can clearly see that the probability of occurrence of the major risk factors and severity. The combination of factor analysis and blind number analysis can effectively quantify operational risk probability of electricity companies to provide guidance and suggestions for planning and construction of power enterprises. 
Power companies must establish a correct sense of risk in operating procedure, including the timely, moderate and appropriate awareness of risk prevention. Timely risk prevention awareness means that the risk exists everywhere at all times, thus risk must run through the whole process of management innovation involving creative generating, design implementation and management operating, every step in the design innovations must take preventive measures to prevent the spread of risk and a chain reaction, rather than re-take remedial action after it occurs; moderate risk prevention awareness means that the risk of management innovation is high and the absolute risk aversion does not exist, so companies cannot blindly follow the trend of innovation and be afraid of innovation in order to avoid losses caused by innovation; appropriate risk prevention awareness means that the idea "retain the large, release the small" also suits for management innovation, and build management innovation safeguards system to ensure that the various processes give support to innovation effort and risk prevention measures implement effectively.

\section{Conclusion}

Risk Quantification of electric power operation system is the basic work of risk management, playing a decisive role for the development of risk budgeting, emergency measures and etc. In the analysis and calculation of the operational risk quantification, there are a lot of blind information, thus taking full advantage of expertise and based on historical data, reduce the risk of models and decisions to make risk assessments objectively reflect the actual situation as much as possible and to provide support for the business operations decision.

The emphasis of this study focuses is that we introduce blind number theory to express risk information that is difficult to describe on the basis of analysis and combing of risk information, combined with the experience of experts to quantify the risk, therefore it is more scientific and objective than traditional methods to reflect the likelihood of risk occurrence. The empirical results show that the blind number method is capable of processing the uncertainties of quantitative assessment of electric power enterprises operational risk and expert information that qualitative assessment methods cannot handle to give the distribution diagram of system reliability indices. It clearly reveals how much the key risks affect the electric power companies, which provides scientific decision basis for the planning and construction of electric power operations.

\section{References}

[1] Jiang Su. Study on Risk Investment Assessment of High-tech Enterprise [J]. Technology Progress and Policy, 2013, 30(9): 5-33.

[2] Hoyt R E , liebenberg A P. The value of enterprise risk management [J]. Journal of Risk and Insurance , $2011,78(4): 795-822$.

[3] Guomai Liu, Lingyu Sun. Research on contractor risk based on factor analysis [J]. Journal of Fujian College of Engineering, 2007, 5(2): 116-120.

[4] Zhuangzhi Tang. Risk And Efficiency of Commercial Banks in China -- Based on Factor Analysis Method [J]. Modern Business Trade Industry, 2009, (6): 62-68.

[5] Lizhong Wang, Junjie Zheng, Lei Zhang. Risk Probability Estimation Method based on Blind Number Theory [J]. Wireless Internet Technology, 2014, (3): 236-240.

[6] Haozhong Cheng, Haifeng Zhu, Jianmin Wang. Flexible Planning of Power Grid Based on Blind Number BM Model [J]. Journal of Shanghai Jiao Tong University, 2003, 37(9): 1347-1350.

[7] Ciwei Gao, Xu Wang. Application of Fuzzy Evaluation Model of Blind Information in Power Grid Planning [J]. Chinese Journal of Electrical Engineering, 2004, 24(9): 24-29. 
[8] Tienan Ma. Evaluation Model of Brand Competitiveness Based on Blind Number Theory [J]. Journal of Sichuan Normal University, 2013, 36(3): 480-484. 